Association for Information Systems

AIS Electronic Library (AISeL)

Wirtschaftsinformatik Proceedings 2005

Wirtschaftsinformatik

February 2005

\title{
Der Einfluss der Spezifität des Humankapitals auf die Frage des Sourcings von Anwendungssoftware- Dienstleistungen
}

Jens Dibbern

Universität Mannheim

Follow this and additional works at: http://aisel.aisnet.org/wi2005

\section{Recommended Citation}

Dibbern, Jens, "Der Einfluss der Spezifität des Humankapitals auf die Frage des Sourcings von Anwendungssoftware-Dienstleistungen" (2005). Wirtschaftsinformatik Proceedings 2005. 23.

http://aisel.aisnet.org/wi2005/23

This material is brought to you by the Wirtschaftsinformatik at AIS Electronic Library (AISeL). It has been accepted for inclusion in Wirtschaftsinformatik Proceedings 2005 by an authorized administrator of AIS Electronic Library (AISeL). For more information, please contact elibrary@aisnet.org. 
In: Ferstl, Otto K, u.a. (Hg) 2005. Wirtschaftsinformatik 2005: eEconomy, eGovernment, eSociety; 7. Internationale Tagung Wirtschaftsinformatik 2005. Heidelberg: Physica-Verlag

ISBN: 3-7908-1574-8

(C) Physica-Verlag Heidelberg 2005 


\title{
Der Einfluss der Spezifität des Humankapitals auf die Frage des Sourcings von Anwendungssoftware-Dienstleistungen
}

\author{
Jens Dibbern \\ Universität Mannheim
}

\begin{abstract}
Zusammenfassung: Seit geraumer Zeit wird die Frage kontrovers diskutiert, unter welchen Umständen es für ein Unternehmen vorteilhaft ist, bestimmte Funktionen der Informationsverarbeitung fremd zu beziehen. Während die einen die Risiken des Outsourcings betonen, indem sie auf den Verlust strategischer Potenziale und erhöhter Transaktionskosten verweisen, sprechen andere von strategischem Outsourcing und hohen Potenzialen der Kosteneinsparung. Der vorliegende Beitrag untersucht die Umstände, unter denen die strategischen Potenziale intern besser ausgeschöpft werden und die Produktions- und Transaktionskosten intern niedriger ausfallen als beim Fremdbezug. Eine zentrale Rolle spielen hierbei das Ausmaß der Spezifität des Humankapitals, Unterschiede in der Vertrauenswürdigkeit und der Motivation zwischen internen und externen Mitarbeitern sowie im Falle des Outsourcings die Höhe der Kapitalbeteiligung am externen Dienstleister. Aus drei komplementären Theorien wird ein Bezugsrahmen entwickelt und auf Basis einer schriftlichen Befragung von 139 deutschen Unternehmen empirisch getestet.
\end{abstract}

Schlüsselwörter: Outsourcingentscheidung, Transaktionskostentheorie, ressourcenorientierter Ansatz, Verfügungsrechte, Theorie der unvollständigen Verträge

\section{Einleitung}

Obwohl Marktforschungsunternehmen ein anhaltendes Wachstum des Outsourcings von Dienstleistungen der betrieblichen Informationsverarbeitung (IV) propagieren, verhalten sich Unternehmen nach wie vor sehr unterschiedlich bei der Frage des Sourcings bzw. der Eigenerstellung oder des Fremdbezugs von Leistungen der IV. Während sich einige Unternehmen für ein Komplettoutsourcing ihrer IV entscheiden, lagern andere nur selektiv bestimmte Teilfunktionen (z. B. den Betrieb des Rechenzentrums) aus [DiHe01; Laci $\left.^{+} 96\right]$. Wieder andere haben eine Bewertung des Fremdbezugs vorgenommen oder Erfahrungen mit der Auslagerung gemacht, sich aber für die Eigenerstellung bzw. die Rückführung (Backsourcing) der IV entschieden [HiLa00]. Zudem gibt es Spezialformen des Outsourcings, wie das Jointventure, bei der eine kapitalmäßige Verflechtung zwischen 
dem Kundenunternehmen und dem externen Dienstleiter besteht oder die Ausgliederung, bei der das Kundenunternehmen eine 100-prozentige Kapitalbeteiligung am externen Dienstleister hält [Hein93].

Dieses sehr unterschiedliche Verhalten von Unternehmen beim Sourcing der IV legt die Vermutung nahe, dass die Sourcingentscheidung situationsbedingt erfolgt. Aus theoretischer Sicht erscheint es daher zweckmäßig, allgemeine, die Entscheidungssituation eines Unternehmens charakterisierende Faktoren herauszuarbeiten, die erklären, warum der Grad des Outsourcings von bestimmten IV-Funktionen variiert. Diesen situativen Ansatz haben in den vergangenen Jahren zahlreiche empirische und konzeptionelle Arbeiten verfolgt [Dibb ${ }^{+}$04]. Ihr Hauptziel bestand darin, die Determinanten des Outsourcings der IV herauszuarbeiten. Am häufigsten wurde hierbei auf die Transaktionskostentheorie (TKT) zurückgegriffen $\left[\mathrm{Dibb}^{+}\right.$04]. Sie liefert eine Entscheidungsgrundlage dafür, unter welchen Umständen die Auslagerung kostengünstiger als die Eigenerstellung ist. Die Umstände beziehen sich dabei auf bestimmte Eigenschaften des Entscheidungsobjekts bzw. der jeweiligen IV-Funktion(en) - insbesondere die Faktorspezifität, die Umweltunsicherheit und die Spezifität des Standorts.

Empirische Untersuchungen über den Einfluss dieser Kontextfaktoren auf die Auslagerung der IV ergaben bislang folgende Ergebnisse (vgl. vor allem [Dibb $\left.{ }^{+} 44\right]$ : Der Einfluss der Umweltunsicherheit konnte kaum nachgewiesen werden. Die Spezifität des Standorts spielt vor allem bei kleinen und mittleren Unternehmen eine Rolle [Dibb ${ }^{+} 03$ ]. Ein Zusammenhang zwischen der Faktorspezifität und dem Grad des Outsourcings konnte insbesondere dann festgestellt werden, wenn auf die Eigenschaften der Humanressourcen und weniger auf technische Besonderheiten abgestellt wurde. Zudem bestätigte es sich nachhaltig, dass der Wunsch nach Kosteneinsparungen zu den wichtigsten Entscheidungskriterien gehört. Besonders aufschlussreich ist in diesem Zusammenhang die Studie von Ang und Straub [AnSt98]. Sie zeigte auf, dass Produktionskosten eine weitaus wichtigere Rolle spielen als Transaktionskosten. Allerdings ist bisher nicht untersucht worden, ob der Einfluss der Faktorspezifität tatsächlich auf Unterschiede in den Transaktions- und Produktionskosten zwischen der Eigen- und Fremdfertigung zurückzuführen ist.

Ausgehend von diesen Beobachtungen verfolgt diese Arbeit das Ziel, die tatsächlichen Gründe für den Einfluss der Spezifität des Humankapitals auf den Grad des Outsourcings näher zu untersuchen. Die Offenlegung der Gründe entspricht der Forderung nach einer Erweiterung der Transaktionstheorie [Burr03]. Um gezielte Aussagen über die selektive Auslagerung von Funktionen der IV zu treffen, bezieht sich die Untersuchung ausschließlich auf das Sourcing von Anwendungssoftware-Dienstleistungen. Sie schließen die Gestaltung und die Wartung von Anwendungssoftware ein. Im folgenden Kapitel wird zunächst der theoretische Bezugsrahmen vorgestellt. Dieser wird im dritten Kapitel operationalisiert und 
empirisch getestet. Abschließend werden die Ergebnisse der Studie diskutiert und ihre Implikationen dargelegt.

\section{Theoretischer Bezugsrahmen}

Der theoretische Bezugsrahmen, ist in Abbildung 1 dargestellt. Die Pfeile verkörpern die einzelne Hypothesen. Die Vorzeichen ,,+“ und ,--“ zeigen an, ob ein positiver oder negativer Zusammenhang zwischen den einzelnen Konstrukten erwartet wird. Von rechts angefangen liest sich die Darstellung wie folgt: Der momentane Grad des Outsourcings der Gestaltung und Wartung von Anwendungssoftware spiegelt sich in der Einstellung des IV-Managements gegenüber der Auslagerung der jeweiligen IV-Funktion wieder (H8). Inwiefern ein Unternehmen eine positive Gesamteinstellung gegenüber dem Outsourcing aufweist und wie hoch der tatsächliche Anteil der Auslagerung der jeweiligen IV-Funktion ausfällt, hängt davon $\mathrm{ab}$, ob die Eigenerstellung gegenüber dem Fremdbezug als kostengünstiger (Produktions- (H2a,b) und Transaktionskosten (H3a,b)) und vorteilhafter bei der Erzielung strategischer Erfolgswirkungen (H6a,b) eingestuft wird. Komparative Vorteile der Eigenerstellung bei diesen drei Zielgrößen ergeben sich insbesondere dann, wenn die für die Gestaltung und Wartung notwendigen Humanressourcen als sehr unternehmensspezifisch eingeschätzt werden (H1a-c).

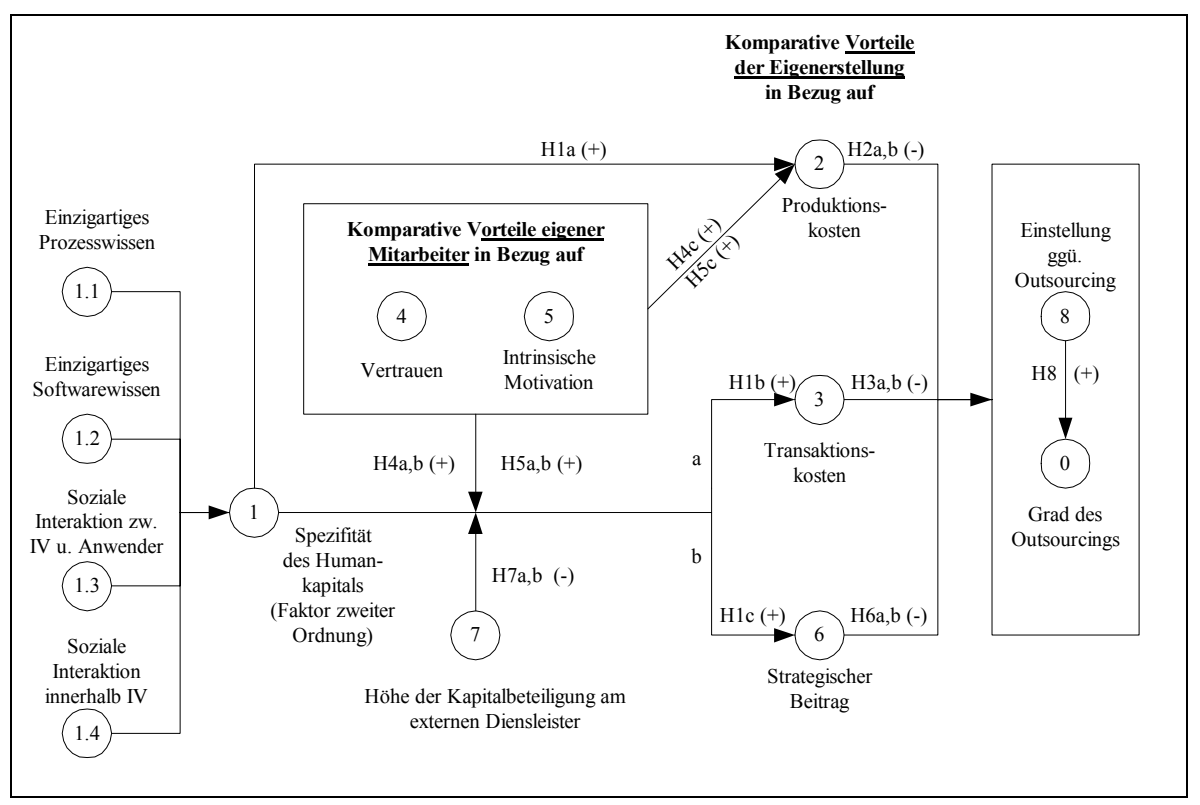

Abbildung 1: Theoretischer Bezugsrahmen 
Dieser Zusammenhang verstärkt sich, wenn interne Mitarbeiter als vertrauenswürdiger und motivierter als externes Personal eingeschätzt werden (H4a,b und H5a,b). Dagegen werden die mit zunehmender Höhe der Humankapitalspezifität eintretenden Nachteile des Fremdbezugs bei den Transaktionskosten und dem Erzielen strategischer Erfolgswirkungen mit steigender Kapitalbeteiligung am externen Dienstleister abgeschwächt $(\mathrm{H} 7 \mathrm{a}, \mathrm{b})$. Zudem wird ein direkter Zusammenhang zwischen internen Produktionskostenvorteilen und Vorteilen in der Vertrauenswürdigkeit (H4c) sowie der Motivation (H5c) der Mitarbeiter postuliert. Im Folgenden werden die einzelnen Zusammenhänge näher erläutert und die Hypothesen abgeleitet.

\subsection{Transaktionskostentheorie}

Einfluss der Spezifität des Humankapitals. Aus Sicht der TKT stellen Unterschiede in den Produktions- und Transaktionskosten zwischen der Eigenerstellung und dem Fremdbezug zwei zentrale Bewertungskriterien der Sourcingentscheidung dar [Will81]. Transaktionskosten umfassen alle Kosten, die im Rahmen der Delegation einer IV-Funktion entstehen. Mit der Delegation verbundene Aufgaben umfassen z. B. die Auswahl des Aufgabenträgers, die Vereinbarung über den Leistungsaustausch, die Überwachung der Leistungserbringung und die mögliche Auflösung der Vertragsbeziehung [PiMa92]. Diese Tätigkeiten dienen dazu, die Gefahr zu verringern, dass sich der Vertragspartner opportunistisch verhält, indem er z. B. wissentlich überhöhte Preise in Rechnung stellt oder qualitativ mindere Leistung erbringt.

Die Transaktionskosten fallen um so höher aus, je geringer die Kenntnisse der Auftraggeber über den Gegenstand und die Art und Weise der Leistungserbringung ausfallen. Dies ist insbesondere dann der Fall, wenn der Prozess der Gestaltung und Wartung von Anwendungssoftware einen hochspezifischen Faktoreinsatz erfordert. Bei der Anwendungsgestaltung und Wartung handelt es sich in erster Linie um Wissensarbeit, d. h. das Wissen der am Arbeitsprozess beteiligten Personen stellt den entscheidenden Produktionsfaktor dar. Die Spezifität des Wissens lässt sich einerseits durch die Art des Wissens und anderseits durch den Prozess, durch den dieses Wissen geschaffen wird, charakterisieren. Als Wissensarten können das Wissen über den Anwendungskontext (bzw. die Unternehmensprozesse) und über die softwaretechnische Realisierung unterschieden werden (basierend auf [Ivia $\left.{ }^{+} 02\right]$ ). Diese beiden Wissensarten können dann als unternehmensspezifisch eingestuft werden, wenn es sich um Wissen über einzigartige Geschäftsprozesse und speziell auf ein Unternehmen zugeschnittene Anwendungssoftware handelt. Es handelt sich de facto um implizites Wissen, dass vor allem durch einen Prozess der sozialen Interaktion der am Gestaltungs- und Wartungsprozess beteiligten Personen entsteht - und zwar einerseits zwischen den IV-Fachleuten (z. B. Systemanalysten, Designer und Programmierer), und anderseits zwischen dem IV- 
Fachpersonal und den Anwendern. Die Spezifität des Humankapitals setzt sich also aus vier Komponenten zusammen (siehe Abbildung 1).

Nach der TKT kann der Gefahr des opportunistischen Verhaltens, die bei der Delegation von hochspezifischen IV-Funktionen besonders hoch ausfällt, intern durch das Autoritätsprinzip effizienter, d. h. mit geringeren Transaktionskosten, begegnet werden.

Gleichzeitig ist zu erwarten, dass mit steigender Humankapitalspezifität die Produktionskosten bei der Eigenerstellung niedriger ausfallen. Produktionskosten sind alle Kosten, die im Rahmen der Verrichtung der eigentlichen Tätigkeiten anfallen, die für die Aufgabenerfüllung einer IV-Funktion notwendig sind. Für externe Dienstleister erfordert die Bereitstellung unternehmensspezifischer Anwendungssoftware einen erheblichen Lernaufwand [BeWa98]. Skaleneffekte mit anderen Kundenunternehmen lassen sich dabei nur bedingt erzielen. Interne IVAbteilungen können dagegen in der Regel auf langjährige Erfahrungen mit den Anforderungen der Anwender und den technischen Eigenschaften der Anwendungssoftware zurückgreifen [Dibb ${ }^{+} 03$ ]. Dies führt zu folgenden Hypothesen:

H la und b: Je spezifischer das erforderliche Humankapital für die Verrichtung einer IVFunktion ausfällt, desto geringer fallen die internen (a) Produktions- und (b) Transaktionskosten gegenüber dem Fremdbezug aus.

H2a: Je geringer die Produktionskosten der Eigenerstellung gegenüber dem Fremdbezug eingeschätzt werden, desto weniger wird eine IV-Funktion ausgelagert.

H3a: Je geringer die Transaktionskosten der Eigenerstellung gegenüber dem Fremdbezug eingeschätzt werden, desto weniger wird eine IV-Funktion ausgelagert.

Antipoden des opportunistischen Verhaltens als Moderatoren. Die Annahme, dass sich bestimmte Akteure opportunistisch verhalten und dass dieses Verhalten intern durch das Autoritätsprinzip effizienter unterbunden werden kann, wird in zunehmenden Maße kritisch hinterfragt [GoMo96]. Es lässt sich argumentieren, dass es neben der Ausübung von Weisungsbefugnissen andere Mechanismen gibt, welche die Gefahr des opportunistischen Verhaltens begrenzen, ohne zu erhöhten Transaktionskosten zu führen. Eine Reihe von Wissenschaftlern vertritt den Standpunkt, dass die Einbeziehung des Konstrukts des Vertrauens den Erklärungsgehalt der TKT erhöhen kann [z. B. GoMo96]. Der Aufbau vertrauensbasierter Beziehungen kann die Gefahr des opportunistischen Verhaltens begrenzen. Dies gilt sowohl für innerbetriebliche als auch für zwischenbetriebliche Beziehungen. Für die Sourcingentscheidung bedeutet dies, dass es bei der Verrichtung von hochspezifischen IV-Funktionen darauf ankommt, ob die internen Mitarbeiter oder das Personal externer Dienstleister als vertrauenswürdiger eingestuft werden. Gelingt es, eine vertrauensbasierte Beziehung mit einem externen Dienstleister aufzubauen, können auch spezifische Aufgaben ausgelagert werden, ohne zu Transaktionskostennachteilen zu führen. Wird hingegen die Vertrauenswürdigkeit des internen Personals höher eingeschätzt, so verstärken sich die internen Transaktionskostenvorteile 
mit zunehmender Spezifität der Anwendungssoftware-Dienstleistungen. Dies führt zu folgender Hypothese:

H 4a: Je vertrauenswürdiger die internen Mitarbeiter verglichen mit dem Personal externer Dienstleister eingeschätzt werden, desto stärker fällt der Zusammenhang zwischen dem Grad der Spezifität des Humankapitals und internen Transaktionskostenvorteilen aus.

Als weiterer Faktor, der opportunistischem Verhalten entgegenwirkt, ohne zu erhöhten Transaktionskosten zu führen, ist die intrinsische Motivation eines Mitarbeiters zu nennen. Mitarbeiter sind dann intrinsisch motiviert, wenn sie bestimmte Tätigkeiten um ihrer selbst Willen ausführen [FrOs97, S. 308]. Im Gegensatz dazu sind Mitarbeiter extrinsisch motiviert, wenn sie allein durch externe Anreize, z. B. durch die Höhe der Leistungsvergütung, motiviert sind. Opportunistisches Verhalten zielt darauf $a b$, sich eigene Vorteile durch die bewusste Inkaufnahme der Schlechterstellung des Vertragspartners zu verschaffen. Insofern ist opportunistisches Verhalten primär extrinsisch motiviert. Je stärker Mitarbeiter bei ihrer Arbeit intrinsisch motiviert sind, desto weniger sprechen sie auf externe Anreize an und desto weniger werden sie sich opportunistisch verhalten. Bei der Anwendungsentwicklung und -wartung hat das soziale Umfeld der Arbeitsverrichtung einen hohen Einfluss auf die Höhe der intrinsischen Motivation [CoCo85]. Gerade das soziale Umfeld erfährt im Falle des Wechsels von der Eigen- zur Fremderstellung (oder umgekehrt) allerdings starke Veränderungen. Die intrinsische Motivation kann sich hierdurch sowohl zum Positiven also auch zum Negativen entwickeln. Es kommt also wiederum darauf an, ob die intrinsische Motivation der internen Mitarbeiter oder die des externen Personals als höher eingeschätzt wird, um zu beurteilen, welche Organisationsform bei spezifischen AnwendungssoftwareDienstleistungen die geringeren Transaktionskosten verspricht. Entsprechend lässt sich folgende Hypothese aufstellen:

H 5a: Je höher die intrinsische Motivation der internen Mitarbeiter im Vergleich zu externem Personal eingeschätzt wird, desto stärker fällt der Zusammenhang zwischen dem Grad der Spezifität des Humankapitals und internen Transaktionskostenvorteilen aus.

Gleichzeitig lässt sich aber auch anführen, dass stärker motivierte Mitarbeiter eine höhere Leistungsbereitschaft zeigen, was sich in einer höheren Produktivität äuBert. Dies trifft auch auf die Gestaltung und Wartung von Anwendungssoftware zu [CoCo85], so dass gefolgert werden kann, dass sich Unterschiede in der intrinsischen Motivation zwischen internen Mitarbeitern und externem Personal auch direkt auf Produktionskostenunterschiede auswirken. Ähnliches gilt für die Vertrauenswürdigkeit. Mitarbeiter müssen sich in der Regel das Vertrauen ihrer Vorgesetzten erarbeiten. Eine hohe Produktivität eines Mitarbeiters stärkt das Vertrauen in ihn. Insofern ist davon auszugehen, dass Unterschiede in der Vertrauenswürdigkeit zwischen internem und externem Personal in direktem Zusammenhang mit den wahrgenommen Produktionskostenunterschieden stehen: 
H 4c: Je vertrauenswürdiger die internen Mitarbeiter gegenüber dem Personal externer Dienstleister eingeschätzt werden, desto höher fallen die internen Produktionskostenvorteile aus.

H 5c: Je höher die intrinsische Motivation der internen Mitarbeiter gegenüber dem Personal externer Dienstleister eingeschätzt wird, desto höher fallen die internen Produktionskostenvorteile aus.

\subsection{Ressourcenorientierter Ansatz}

Unternehmen, welche die Sourcingentscheidung allein vor dem Hintergrund der Kostenminimierung treffen, laufen jedoch Gefahr, die durch die IV erzielten Erfolgswirkungen zu vernachlässigen. Nicht nur die Kosten der Verrichtung einer IV-Funktion können zwischen der Eigenerstellung und dem Fremdbezug variieren sondern auch die durch sie induzierten Erfolgswirkungen. Diese Sichtweise lässt sich auf den ressourcenorientierten Ansatz (ROA) stützen. Er geht davon aus, dass Unternehmen unterschiedliche Ressourcen und Fähigkeiten aufweisen, und dass diese Unterschiede die Grundlage für das Erzielen von Wettbewerbsvorteilen gegenüber Konkurrenten darstellen [Barn91].

Nach dem ROA kann die IV dann als strategisch eingestuft werden, wenn sie (1) einen Beitrag zum Unternehmensgewinn liefert, und wenn die für diesen Erfolgsbeitrag erforderlichen Ressourcen und Fähigkeiten (2) nicht imitierbar, (3) nicht substituierbar (4) und nicht am Markt handelbar sind [Barn91; ClRo91].

Der Beitrag zum Unternehmensgewinn erfolgt durch die IV in der Regel indirekt [ClRo91]. Ihr wird die Rolle komplementärer strategischer Ressourcen und Fähigkeiten zugeschrieben [ClRo91, S. 280ff.], d. h. sie kann durch die Automatisierung und verbesserte Informationsversorgung in den primären Geschäftsprozessen bzw. Betriebsfunktionen zur Kostensenkung und/oder Leistungssteigerung beitragen. Dies gilt auch für Teilfunktionen der IV, wie die Anwendungsgestaltung und -wartung. Gerade dem Anwendungsportfolio eines Unternehmens wird bei der strategischen Planung der IV eine wichtige Rolle zugesprochen [Ragh99].

Die dazu erforderlichen Ressourcen und Fähigkeiten können insbesondere dann als nicht imitierbar und substituierbar eingestuft werden, wenn sie unternehmensspezifisch sind. Es besteht also ein direkter Zusammenhang zwischen der Faktorspezifität aus der TKT und dem Konzept der spezifischen Ressourcen aus dem ROA [Dibb $\left.{ }^{+} 01\right]$.

Es stellt sich allerdings die Frage, ob ein externer Dienstleister bereit ist, derartige spezifische Investitionen zu tätigen. Ein externer Dienstleister verfolgt seine eigenen strategischen Ziele. Sein Unternehmensgewinn lässt sich insbesondere dann erhöhen, wenn sich bei der Erbringung von Anwendungssoftware-Dienstleistungen Skalenerträge durch den Einsatz bei mehreren Kunden erzielen lassen. Gerade dies ist bei unternehmensspezifischen Investitionen allerdings nicht der 
Fall. Insofern besteht auch hier - in Analogie zur TKT - die Gefahr des opportunistischen Verhaltens des externen Dienstleisters. Dies bedeutet, dass es auch hier wichtig ist, wie die Vertrauenswürdigkeit und die intrinsische Motivation des Personals des externen Dienstleisters gegenüber den eigenen Mitarbeitern eingeschätzt wird. Interne Vertrauens- und Motivationsvorteile können die Wahrnehmung interner Vorteile in der Ausschöpfung von strategischen Potenzialen spezifischer Investitionen verstärken. Werden dagegen Verhaltensvorteile beim externen Personal gesehen, begünstigt dies die Bildung strategischer Allianzen. Diese Zusammenhänge lassen sich in den folgenden beiden Hypothesen zusammenfassen:

H 1c: Je spezifischer das erforderliche Humankapital für die Verrichtung von Anwendungssoftware-Dienstleistungen ausfällt, desto stärker wird der strategische Beitrag einer IV-Funktion durch die Auslagerung gefährdet.

H 4b: Je vertrauenswürdiger die internen Mitarbeiter verglichen mit dem Personal externer Dienstleister eingeschätzt werden, desto stärker fällt der Zusammenhang zwischen dem Grad der Humankapitalspezifität und internen Vorteilen bei der Erzielung strategischer Erfolgswirkungen einer IV-Funktion aus.

H 5b: Je höher die intrinsische Motivation der internen Mitarbeiter im Vergleich zu externem Personal eingeschätzt wird, desto stärker fällt der Zusammenhang zwischen dem Grad der Humankapitalspezifität und internen Vorteilen bei der Erzielung strategischer Erfolgswirkungen einer IV-Funktion aus.

H 6a: Je höher die internen Vorteile des Beitrags einer IV-Funktion zur strategischen Zielerreichung eines Unternehmens eingestuft werden, desto weniger wird diese Funktion ausgelagert.

\subsection{Theorie der unvollständigen Verträge}

Ein interessanter Unterschied zwischen der TKT und dem ROA besteht in der Rolle unvollständiger Verträge. Nach der TKT können bei der Delegation von Aufgaben, die hochspezifisches Wissen erfordern, nur unvollständige Verträge über deren Verrichtung geschlossen werden. Dies erhöht die Gefahr opportunistischen Verhaltens und zieht erhöhte Transaktionskosten nach sich. Hingegen postuliert der ROA, dass Situationen, in denen Verträge nur unvollständig geschlossen werden können, die Grundvoraussetzung für das Erzielen von Wettbewerbsvorteilen gegenüber Konkurrenten sind. Die Herausforderung besteht demnach darin, die positiven Erfolgswirkungen spezifischer Investitionen aufrechtzuerhalten, ohne gleichzeitig erhöhte Transaktionskosten in Kauf nehmen zu müssen. Zwei mögliche verhaltensbezogene Sicherungsmechanismen wurden bereits angesprochen. Es handelt sich um die Schaffung vertrauensbasierter Beziehungen und die Förderung der intrinsischen Motivation der IV-Mitarbeiter. Eine dritte Möglichkeit besteht nach der originären TKT darin, opportunistisches Verhalten durch die Ausübung von Autorität zu begrenzen. Es wird angenommen, dass das hierar- 
chische System innerhalb eines Unternehmens hierfür bessere Voraussetzungen schaffe als eine Beziehung mit einem externen Dienstleister [Will81].

Die letztere Annahme hat bei den Vertretern der Lehre von den Verfügungsrechten (,property rights theory“) vehemente Kritik ausgelöst. Aus ihrer Sicht gibt es keinen Grund, warum die Weisungsbefugnisse gegenüber Angestellten höher ausfallen sollten als gegenüber einem externen Dienstleister [AlDe72, S. 777]. In beiden Fällen handele es sich lediglich um unterschiedliche vertragliche Vereinbarungen. Diese Kontoverse haben Vertreter der Theorie unvollständiger Verträge veranlasst, die Frage zu stellen, worin überhaupt Autorität begründet sei. Die Antwort liegt aus ihrer Sicht in den Eigentumsrechten begründet [GrHa86]. Der Eigentümer der Produktionsfaktoren, die für die Verrichtung einer Aufgabe notwendig sind, hat das Recht, über die Verteilung der resultierenden Erträge zu entscheiden. Innerhalb eines Unternehmens obliegen die Eigentumsrechte in der Regel den Kapitaleignern. Diese wiederum übertragen ihre Weisungsbefugnisse auf das Management der Unternehmung. Das Management entscheidet über die Verteilung eines Teils der Erträge und somit auch über die Beschäftigung. Verhalten sich IV-Fachkräfte opportunistisch, so kann sie das Management auf Basis von Eigentumsrechten entlassen. Im Kontext der Gestaltung und Wartung von Anwendungssoftware bedeutet dies, dass ein Unternehmen dann die Gefahr opportunistischen Verhaltens eindämmen kann, wenn es Eigentümer der rechtlichen Institution ist, welche die IV-Fachkräfte beschäftigt. Natürlich kann auch der Vertrag mit einem externen Anbieter aufgelöst werden. Allerdings bedeutet dies nicht automatisch, dass dessen Mitarbeiter ihre Beschäftigung verlieren. Bei rein vertraglich basierten Outsourcingbeziehungen hat ein Unternehmen keine direkten Durchgriffsrechte auf einzelne Mitarbeiter des externen Dienstleisters. Anders verhält es sich, wenn das Unternehmen eine Kapitalbeteiligung am externen Dienstleister hält. Durch Kapitalbeteiligungen werden Eigentumsrechte übertragen. Kapitalbeteiligungen am externen Dienstleister können daher sowohl interne Transaktionskostenvorteile vermindern als auch die strategischen Risken der Auslagerung begrenzen. Dies führt zu folgenden Hypothesen:

H 7a und b: Je höher die Kapitalbeteiligung am externen Dienstleister ist, desto weniger führt eine erhöhte Spezifität des Humankapitals zu (a) internen Transaktionskostenvorteilen und (b) Vorteilen bei den strategischen Erfolgswirkungen der jeweiligen IV-Funktion.

\subsection{Einstellungen versus momentanes Verhalten}

Die bisherigen Überlegungen gehen davon aus, dass sich die wahrgenommenen Unterschiede zwischen der Eigenerstellung und dem Fremdbezug bei den Produktionskosten, den Transaktionskosten und den strategischen Erfolgswirkungen direkt im Grad des Outsourcings widerspiegeln. Entscheidungstheoretisch setzt dies voraus, dass jede Wahrnehmungsänderung des Managements in Bezug auf diese Zielgrößen eine sofortige Anpassung des Sourcingverhaltens nach sich zieht. In der Realität ist jedoch davon auszugehen, dass dieser Anpassungsprozess mit Ver- 
zögerung verläuft und dass zudem verschiedene institutionelle Rahmenbedingungen und sonstige Einflüsse einem streng rationalen Verhalten des Managements im Wege stehen können. Es erscheint daher zweckmäßig, zu untersuchen, inwieweit diese drei komparativen Zielgrößen die Gesamteinstellung der obersten Leitungsinstanz der IV gegenüber dem Outsourcing einer IV-Funktion beeinflussen und wie sehr diese Gesamteinstellung mit dem momentanen Auslagerungsverhalten korrespondiert. Entsprechend lassen sich folgende Hypothesen aufstellen:

H 5b-7b: Wahrgenommene Unterschiede zwischen der Eigenerstellung und dem Fremdbezug bei (5b) den Produktionskosten, (6b) den Transaktionskosten und (7b) dem strategischen Beitrag wirken nachhaltig auf die Gesamteinstellung des Managements gegenüber dem Fremdbezug einer IV-Funktion.

H8: Je positiver die Gesamteinstellung gegenüber der Auslagerung einer IV-Funktion ausfällt, desto höher fällt der Grad der Auslagerung dieser Funktion aus.

\section{Empirische Untersuchung}

Um die Validität des theoretischen Bezugsrahmens zu überprüfen, wurden mit Hilfe eines standardisierten Fragebogens umfangreiche Daten aus der Unternehmenspraxis erhoben. Die Gestaltung des Erhebungsinstrumentariums erfolgte unter Berücksichtigung der Anforderungen der Kausalanalyse. Die Anwendung der Kausalanalyse entspricht in besonderem Maße dem konfirmatorischen Charakter der Studie, da sie sowohl den Test einer Vielzahl von Hypothesen als auch die Überprüfung der Güte und Zuverlässigkeit des Erhebungsinstrumentariums erlaubt.

\subsection{Operationalisierung der Konstrukte}

Zunächst galt es, die einzelnen Konstrukte des Bezugsrahmens messbar zu machen. Die Variablenmessung erfolgte unter Berücksichtigung der Anforderungen der Kausalanalyse. Es wurden ausschließlich reflektive Indikatorblöcke mit mindesten zwei Messindikatoren (Fragen) pro Konstrukt gebildet [Chin98]. Nach Möglichkeit wurden die Messindikatoren aus früheren empirischen Untersuchungen übernommen oder abgeleitet. In Tabelle 1 findet sich jeweils ein Beispielindikator pro Konstrukt sowie die dazugehörige Literaturquelle. Als Messskala wurde in den meisten Fällen eine Likert-Skala mit 5 Punkten verwendet, die von ,$+2=$ trifft voll zu“ bis ,, $2=$ trifft gar nicht zu“ reichte, mit, $0=$ teils - teils“" als Mittelpunkt. Abweichungen von dieser Skala sind in Tabelle 1 kenntlich gemacht (z. B. die Verwendung von Prozentangaben). Jede einzelne Frage bzw. jedes einzelne Statement wurde einerseits auf die Gestaltung und anderseits auf die Wartung von Anwendungssoftware bezogen. 


\begin{tabular}{|c|c|c|c|}
\hline Nr. & Konstrukt & Quelle & Beispielfrage [Gesamtanzahl] \\
\hline 0 & $\begin{array}{l}\text { Grad des Outsour- } \\
\text { cings }\end{array}$ & $\begin{array}{l}\text { Basiert auf } \\
{[\text { DiHe01] }}\end{array}$ & $\begin{array}{l}\text { Bitte schätzen Sie ... den durchschnittlichen gegenwärtigen } \\
\text { prozentualen Anteil (von } 0 \text { bis } 100 \% \text { ) des Outsourcings ... } \\
\text { bezogen auf ... das Gesamtbudget der IV-Funktion. [3] }\end{array}$ \\
\hline 1.1 & $\begin{array}{l}\text { Einzigartiges Pro- } \\
\text { zesswissen }\end{array}$ & $\begin{array}{l}\text { Basiert auf } \\
{[\mathrm{AnCu} 97]}\end{array}$ & $\begin{array}{l}\text { Die Verrichtung der eigentlichen Arbeiten in der jeweili- } \\
\text { gen IV-Funktion verlangt .... ein gutes Verständnis über } \\
\text { die besonderen Geschäftsprozesse in Ihrer Organisation. } \\
\text { [2] }\end{array}$ \\
\hline 1.2 & $\begin{array}{l}\text { Einzigartiges Soft- } \\
\text { warewissen }\end{array}$ & $\begin{array}{l}\text { Basiert auf } \\
{[\mathrm{AnCu} 97]}\end{array}$ & $\begin{array}{l}\text {... detailliertes Wissen über Softwaresysteme, die speziell } \\
\text { auf die Bedürfnisse Ihrer Organisation zugeschnitten sind. } \\
{[2]}\end{array}$ \\
\hline 1.3 & $\begin{array}{l}\text { Soziale Interaktion } \\
\text { zw. IV u. Anwender }\end{array}$ & $\begin{array}{l}\text { Neukonzep- } \\
\text { tion basiert } \\
\left.\text { auf [Pint }{ }^{+} 93\right]\end{array}$ & $\begin{array}{l}\text { „Bei der Verrichtung ... der jeweiligen IV-Funktion } \\
\text { ist es wichtig, ausgeprägte soziale / interpersonale Ar- } \\
\text { beitsbeziehungen ... zwischen Aufgabenträgern in der IV } \\
\text { und Anwendern / Auftraggebern zu pflegen. [2] }\end{array}$ \\
\hline 1.4 & $\begin{array}{l}\text { Interaktion } \\
\text { lb IV }\end{array}$ & 1.3 & $\begin{array}{l}\text {... innerhalb der Gruppe von IV-Aufgabenträgern zu pfle- } \\
\text { gen. [2] }\end{array}$ \\
\hline 2 & $\begin{array}{l}\text { Produkti- } \\
\text { tenvorteile }\end{array}$ & $\begin{array}{l}\text { Basiert auf } \\
{[\text { AnSt98] }}\end{array}$ & $\begin{array}{l}\text { „...arbeitet unser internes Personal kosteneffizienter als ein } \\
\text { externer Dienstleister.“ [5] }\end{array}$ \\
\hline 3 & $\begin{array}{l}\text { erne Transakti- } \\
\text { skostenvorteile }\end{array}$ & $\begin{array}{l}\text { Basiert auf } \\
\text { [AnSt98] }\end{array}$ & $\begin{array}{l}\text {,... fallen bei interner Leistungserstellung geringere } \\
\text { Verhandlungs-, Management- und Koordinationskosten an } \\
\text { als beim Fremdbezug.“ }[4]\end{array}$ \\
\hline 4 & Vertr & uf & $\begin{array}{l}\text { „Wie viel mehr oder weniger haben Sie bei Ihren eigenen } \\
\text { Mitarbeitern im Vergleich zum Personal externer } \\
\text { Dienstleister den Glauben und das Gefühl, dass sie die } \\
\text { Aufgaben auch dann richtig ausführen, wenn die Möglich- } \\
\text { keit besteht, sich opportunistisch zu verhalten?“ [3] }\end{array}$ \\
\hline 5 & $\begin{array}{l}\text { rinsische Motiva- } \\
\mathrm{n}\end{array}$ & $\begin{array}{l}\text { Neukonzep- } \\
\text { tion basiert } \\
\text { auf [CaSt75] }\end{array}$ & $\begin{array}{l}\text { „Unter Nichtberücksichtigung von solchen Anreizen zur } \\
\text { Förderung der Leistungsbereitschaft, die zu direkten geld- } \\
\text { werten Vorteilen führen (z. B. Zusatzprämien), sind unsere } \\
\text { eigenen Mitarbeiter gegenüber dem Personal externer } \\
\text { Dienstleister viel motivierter, hochwertige Arbeit in der } \\
\text { IV-Funktion zu verrichten.“ [3] }\end{array}$ \\
\hline 6 & $\begin{array}{l}\text { Interne Vorteile bei } \\
\text { strategischen Er- } \\
\text { folgswirkungen } \\
\end{array}$ & $\begin{array}{l}\text { Neukonzep- } \\
\text { tion basiert } \\
\text { auf [Barn91] } \\
\end{array}$ & $\begin{array}{l}\text { „Der zentrale Beitrag dieser IV-Funktion zum Erzielen } \\
\text { von Wettbewerbsvorteilen wird geschwächt, wenn sie } \\
\text { nicht eigenerstellt, sondern fremd bezogen wird.“ [3] }\end{array}$ \\
\hline 7 & $\begin{array}{l}\text { Höhe der Kapital- } \\
\text { beteiligung am ex- } \\
\text { ternen Dienstleister }\end{array}$ & $\begin{array}{l}\text { Basiert auf } \\
\text { [Hein93] }\end{array}$ & $\begin{array}{l}\text { „... wieviel Prozent des Kapitals der externen Dienstleister } \\
\text { Ihre Organisation durchschnittlich hält.“ [1]. }\end{array}$ \\
\hline 8 & $\begin{array}{l}\text { Einstellung zum } \\
\text { Outsourcing }\end{array}$ & $\begin{array}{l}\text { Basiert auf } \\
{[\mathrm{AjFi} 80]}\end{array}$ & $\begin{array}{l}\text { „Insgesamt gesehen, ist die Wahl, Aufgaben in dieser IV- } \\
\text { Funktion von einem externen Dienstleister verrichten zu } \\
\text { lassen, schlecht/gut (-3 to +3).“ }[6]\end{array}$ \\
\hline
\end{tabular}

Tabelle 1: Messung der Konstrukte

\subsection{Datenerhebung}

Der Fragebogen wurde zunächst anhand von Praktikern auf seine inhaltliche Verständlichkeit geprüft und anschließend an 916 Unternehmen in Deutschland (516 im Maschinenbau und 400 im Finanzwesen) verschickt. Als Adressat des Fragebogens wurde jeweils namentlich die oberste Leitungsinstanz der Informations- 
verarbeitung angesprochen. Es wurden lediglich Unternehmen mit mehr als 500 Mitarbeitern berücksichtigt. Insgesamt wurden 139 auswertbare Fragebögen zurückgesandt, was einer Rücklaufquote von 15,2\% entspricht. Aufgrund der Tatsache, dass die Daten sowohl für die Gestaltung als auch für die Wartung erhoben wurden, lagen insgesamt 278 Datensätze für den Modelltest vor.

Um sicherzustellen, dass die auswertbaren Fragebögen eine in sich homogene Teilmenge der Grundgesamtheit darstellen, wurden die sofort antwortenden Unternehmen mit den erst in einer Nachfassaktion antwortenden Unternehmen anhand der Unternehmenscharakteristika Umsatz, Anzahl der Mitarbeiter und Auslagerungsanteil verglichen. Es konnten keine signifikanten Abweichungen zwischen den Gruppen festgestellt werden (t-Test, $p<0,05$ ).

\subsection{Datenanalysemethode}

Für den Modelltest wurde das vorhersageorientierte Verfahren der Kausalanalyse „Partial Least Squares“ (PLS) herangezogen. Dieses Verfahren stellt gegenüber dem kovarianzbasierten Verfahren der Kausalanalyse geringere Anforderungen an das zu Grunde liegende Datenmaterial [Chin98]. Zudem erlaubt es die Spezifikation von Faktoren zweiter Ordnung im sogenannten molaren Modus, welcher der Logik formativer Indikatorblöcke folgt [ChGo95]. Diese Modellierungsform fand beim Konstrukt der Humankapitalspezifität ihre Anwendung. Der Modelltest gliedert sich auf in den Test der Validität der Messgrößen der einzelnen Variablen (Messmodell), den Test der Güte des Gesamtmodells (bei PLS die erklärte Varianz) und den Hypothesentest (Strukturmodell). Signifikanztests wurden mit Hilfe des „bootstrap resampling“-Verfahrens durchgeführt [Chin98]. Die Moderatoreffekte wurden mit Hilfe des ,two-way-interaction“-Verfahrens getestet [Chin $\left.{ }^{+} 03\right]$, d. h. es wurden zunächst Produkte aus den standardisierten Werten der moderierenden Variablen (z. B. Vertrauen) und der unabhängigen Variablen (Humankapitalspezifität) gebildet. Anschließend wurde der direkte Einfluss sowohl des Moderators als auch der unabhängigen Variablen auf die jeweils abhängige Variable (z. B. Transaktionskosten) getestet. Bei einem signifikanten Einfluss der Produktvariable liegt ein Moderatoreffekt vor [Chin ${ }^{+}$3] .

\subsection{Ergebnisse des Modelltests}

\subsubsection{Deskriptive Ergebnisse}

Die deskriptiven Auswertungen beschreiben die Eigenschaften der Stichprobe. Tabelle 2 gibt einen Überblick über die Mittelwerte ausgewählter Beschreibungsvariablen - differenziert nach Branchen und, sofern vorhanden, nach IV-Funktion. Die Auswertungen zeigen auf, dass die antwortenden IV-Verantwortlichen mit durchschnittlich knapp 16 Jahren Unternehmenszugehörigkeit als relativ erfahren 
eingestuft werden können. Zwischen dem Maschinenbau und dem Finanzsektor bestehen einige Unterschiede. So fällt auf, dass im Finanzsektor etwa dreimal soviel Geld für die IV ausgegeben wird und dort nicht nur der Anteil der Auslagerung, sondern auch die durchschnittliche Kapitalbeteilung am externen Dienstleister höher ausfällt. Insgesamt fallen die Ausgaben für die Auslagerung der untersuchten deutschen Unternehmen mit durchschnittlich 41,6 \% für die Gestaltung und 38,7 \% für die Wartung der Anwendungssoftware überraschend hoch aus.

\begin{tabular}{|l|l|c|c|c|c|c|c|}
\hline \multicolumn{2}{|c|}{ Variable } & \multicolumn{2}{c|}{ Maschinenbau } & \multicolumn{2}{c|}{ Finanz } & \multicolumn{2}{c|}{ Gesamt } \\
\cline { 3 - 8 } & $\mathbf{n}$ & Mittelwert & $\mathbf{n}$ & Mittelwert & $\mathbf{n}$ & Mittelwert \\
\hline $\begin{array}{l}\text { Unternehmenszugehörigkeit des IV-Leiters (in } \\
\text { Jahren) }\end{array}$ & 61 & 13,6 & 74 & 17,8 & 135 & 15,9 \\
\hline \multirow{4}{*}{ Anzahl der Mitarbeiter } & Gesamtunternehmen & 58 & 3475 & 67 & 1952 & 125 & 2648 \\
\cline { 2 - 8 } & gesamte IV & 61 & 52 & 73 & 118 & 134 & 88 \\
\cline { 2 - 8 } & Anwendungsgestaltung & 59 & 16 & 73 & 38 & 132 & 29 \\
\cline { 2 - 8 } & Anwendungswartung & 59 & 13 & 72 & 39 & 131 & 27 \\
\hline $\begin{array}{l}\text { Anteil des IV-Budgets am Umsatz } \\
\text { (Maschinenbau) bzw. der Bilanzsumme (Finanz) }\end{array}$ & 56 & $2,1 \%$ & 48 & $6,6 \%$ & 104 & $4,2 \%$ \\
\hline Gesamtumsatz bzw. Bilanzsumme (in Mio. EUR) & 55 & 483,5 & 56 & 6417,3 & 111 & 3477,1 \\
\hline $\begin{array}{l}\text { Anteil des Budget für } \\
\text { Outsourcing }\end{array}$ & Anwendungsgestaltung & 62 & $30,4 \%$ & 77 & $50,6 \%$ & 139 & $41,6 \%$ \\
\cline { 2 - 8 } & Anwendungswartung & 62 & $28,9 \%$ & 77 & $46,6 \%$ & 139 & $38,7 \%$ \\
\hline $\begin{array}{l}\text { Kapitalbeteiligung am } \\
\text { externen Dienstleister }\end{array}$ & Anwendungsgestaltung & 50 & $6,8 \%$ & 59 & $14,0 \%$ & 109 & $10,7 \%$ \\
\cline { 2 - 8 } & Anwendungswartung & 50 & $6,9 \%$ & 59 & $14,3 \%$ & 109 & $10,9 \%$ \\
\hline
\end{tabular}

Tabelle 2: Deskriptive Auswertungen

\subsubsection{Messmodell und erklärte Varianz}

Messvalidität. Die Messgrößen der latenten Variablen erwiesen sich als sehr stabil. Die Indizes für die Indikator- und Konstruktreliabilität übertrafen die geforderten Schwellenwerte in so gut wie allen Fällen. Alle Faktorladungen erwiesen sich als hoch signifikant $(\mathrm{p}<0,001)$ und überstiegen den empfohlenen Wert von 0,7 - bis auf einen Indikator des Vertrauenskonstrukts; er wies einen Wert von 0,61 auf und wurde entfernt. Ein Indikator des Konstrukts Produktionskosten wies einen Wert von 0,67 auf. Er wurde aufgrund der konstant hohen Werte der anderen vier Indikatoren beibehalten. Die durchschnittlich erklärte Varianz der Konstrukte wies in allen Fällen Werte über 0,8 auf (mind. 0,7), die „,composite reliabilty“" war jeweils größer als 0,6 (mind. 0,5).

Erklärte Varianz $\left(R^{2}\right)$. Das Untersuchungsmodell weist zwei zentrale abhängige Variablen auf: (1) den momentanen Grad des Outsourcings der Gestaltung und der Wartung von Anwendungssoftware und (2) die Einstellung gegenüber dem Outsourcing beider IV-Funktionen. Obwohl der Studie nicht der Anspruch zu Grunde lag, die Varianz dieser beiden Faktoren möglichst umfassend zu erklären, sondern vielmehr die unterschiedliche Wirkungsweise der Humankapitalspezifität zu verdeutlichen, fielen die Werte der quadrierten multiplen Korrelation mit 0,26 und 
0,35 ansprechend aus - gleiches galt für die drei komparativen Größen, Produktionskosten, Transaktionskosten und strategischer Beitrag, deren erklärte Varianz zwischen 14 und 20 Prozent liegt (Siehe Abbildung 2).

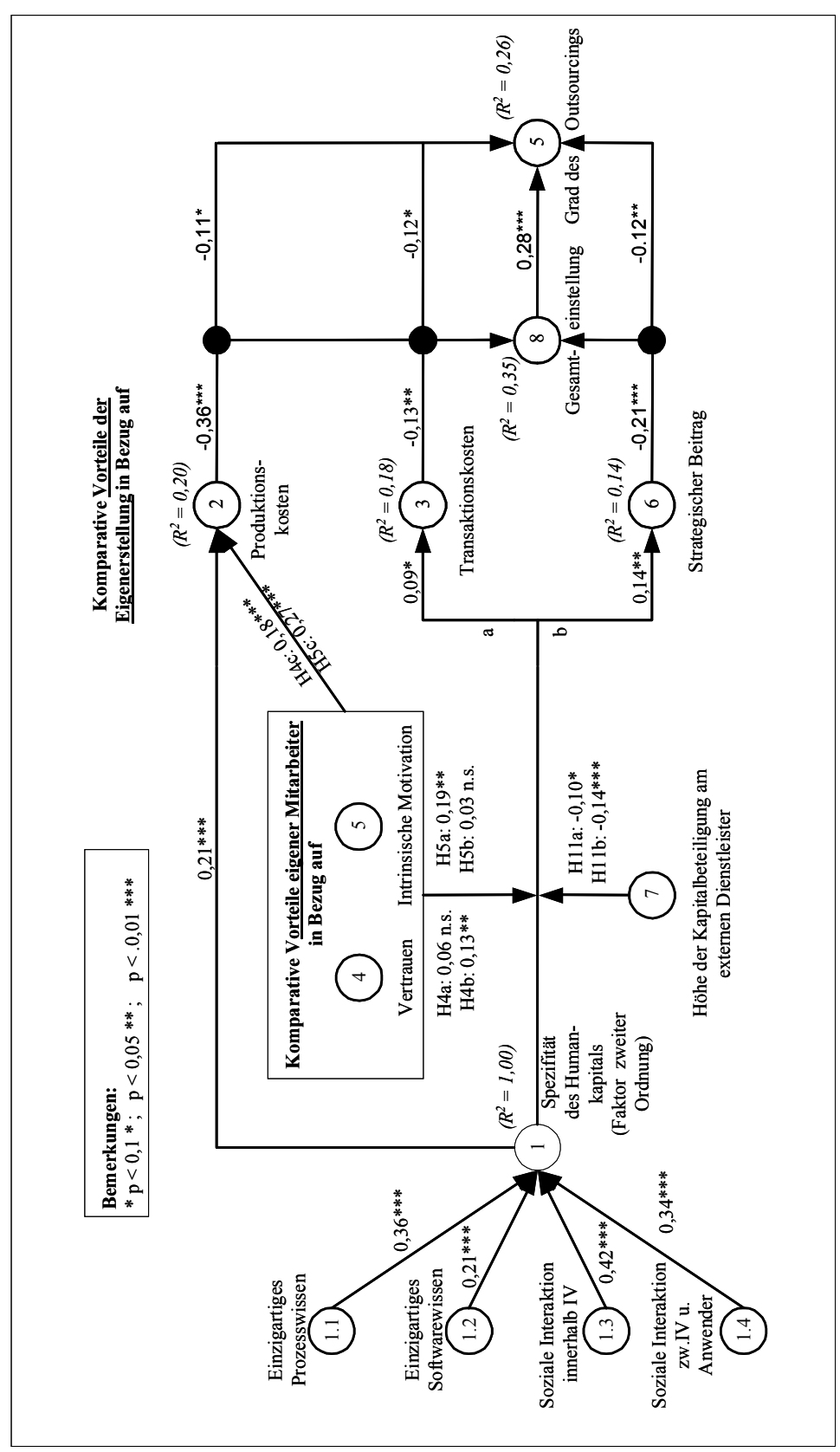

Abbildung 2: Testergebnisse des Strukturmodells 


\subsubsection{Strukturmodell}

Die Ergebnisse des Hypothesentests sind in Abbildung 2 grafisch dargestellt. Die einzelnen Pfadkoeffizienten geben jeweils die Stärke und das Signifikanzniveau ( $\mathrm{t}$-Wert, Student-t-Verteilung, $\mathrm{df}=277$ ) der hypothetisierten Zusammenhänge an. Im Folgenden werden die Ergebnisse differenziert nach direkten Einflüssen und Moderatoreffekten näher beschrieben.

\subsubsection{Direkte Einflüsse}

Faktor zweiter Ordnung: Bestätigt: Die Einflüsse aller vier Faktoren der Humankapitalspezifität (Faktor 2ter Ordnung) sind positiv und signifikant. Einzigartiges Softwarewissen $(0,21, \mathrm{t}=19,7)$ trägt etwas weniger zur Bestimmung der Spezifität des Humankapitals bei als einzigartiges Prozesswissen $(0,36, t=21,1)$, soziale Interaktion innerhalb der IV $(0,42, \mathrm{t}=27,8)$ und soziale Interaktion zwischen IV und Anwendern $(0,35, \mathrm{t}=25,6)$.

Hypothese 1a-c: Bestätigt: Die Pfadkoeffizienten zwischen der Spezifität des Humankapitals und den drei komparativen Vorteilen der Eigenerstellung bei (a) den Produktionskosten $(0,21, \mathrm{t}=3,46)$, (b) den Transaktionskosten $(0,09, \mathrm{t}=1,34)$ und (c) dem strategische Beitrag $(0,14, \mathrm{t}=2,24)$ weisen positive Werte auf und sind signifikant. Der Einfluss auf interne Produktionskostenvorteile fällt am stärksten aus.

Hypothese 2a und b: Bestätigt: Die Pfadkoeffizienten zwischen der Höhe interner Produktionskostenvorteile und der Einstellung gegenüber dem Outsourcing (-0,36, $\mathrm{t}=5,00)$ sowie dem Grad des Outsourcings $(-0,11, \mathrm{t}=1,32)$ weisen jeweils einen negativen Wert auf und sind signifikant. Der Einfluss auf die Einstellungen fällt deutlich stärker aus.

Hypothese 3a und b: Bestätigt: Die Pfadkoeffizienten zwischen der Höhe interner Transaktionskostenvorteile und der Einstellung gegenüber dem Outsourcing $(-0,13, \mathrm{t}=1,91)$ sowie dem Grad des Outsourcings $(-0,12, \mathrm{t}=1,61)$ weisen jeweils einen negativen Wert auf und sind signifikant.

Hypothese 6a und b: Bestätigt: Die Pfadkoeffizienten zwischen der Höhe interner Vorteile beim strategischen Beitrag und der Einstellung gegenüber dem Outsourcing $(-0,21, \mathrm{t}=3,83)$ sowie dem Grad des Outsourcings $(-0,12, \mathrm{t}=1,84)$ weisen jeweils einen negativen Wert auf und sind signifikant. Der Einfluss auf die Einstellungen fällt deutlich stärker aus.

Hypothese 4c: Bestätigt: Der Pfadkoeffizient zwischen relativen Vorteilen eigener Mitarbeiter in Bezug auf ihre Vertrauenswürdigkeit und relativen internen Produktionskostenvorteilen $(0,18, \mathrm{t}=2,72)$ weist einen positiven Wert auf und ist signifikant.

Hypothese 5c: Bestätigt: Der Pfadkoeffizient zwischen relativen Vorteilen eigener Mitarbeiter in Bezug auf ihre intrinsische Motivation und relativen internen 
Produktionskostenvorteilen $(0,26, \mathrm{t}=3,35)$ weist einen positiven Wert auf und ist signifikant.

Hypothese 8: Bestätigt: Der Pfadkoeffizient zwischen der Einstellung gegenüber dem Outsourcing und dem Grad des Outsourcings ist positiv und signifikant $(0,28, \mathrm{t}=3,85)$.

\subsubsection{Moderatoreffekte}

Hypothese 4a: Abgelehnt: Anders als erwartet weist der Pfadkoeffizient zwischen dem Produkt Humankapitalspezifität $x$ Vertrauen und der Höhe interner Transaktionskostenvorteile $(-0,06, \mathrm{t}=0,73)$ ein negatives statt positives Vorzeichen auf und er ist nicht signifikant.

Hypothese 4b: Bestätigt: Der Pfadkoeffizient zwischen dem Produkt Humankapitalspezifität $x$ Vertrauen und der Höhe interner Vorteile bei der Generierung strategischer Erfolgswirkungen $(0,12, \mathrm{t}=1,90)$ ist positiv und signifikant.

Hypothese 5a: Bestätigt: Der Pfadkoeffizient zwischen dem Produkt Humankapitalspezifität $x$ intrinsische Motivation und der Höhe interner Transaktionskostenvorteile $(0,18, \mathrm{t}=1,75)$ ist positiv und signifikant.

Hypothese 5b: Abgelehnt: Der Pfadkoeffizient zwischen dem Produkt Humankapitalspezifität $x$ intrinsische Motivation und der Höhe interner Vorteile bei der Generierung strategischer Erfolgswirkungen $(0,03, \mathrm{t}=0,47)$ ist vernachlässigbar gering und nicht signifikant.

Hypothese 7a und b: Bestätigt: Der Koeffizient zwischen dem Produkt Humankapitalspezifität $x$ Kapitalbeteiligung am Dienstleister und der Höhe interner Transaktionskostenvorteile $(-0,10, \mathrm{t}=1,53)$ sowie interner Vorteile bei der Generierung strategischer Erfolgswirkungen $(-0,14, \mathrm{t}=2,7)$ ist negativ und signifikant.

\section{Diskussion der Ergebnisse und Implikationen}

Insgesamt hat sich der theoretische Bezugsrahmen als relativ robust gegenüber dem Versuch der empirischen Falsifikation erwiesen. Allein zwei der Moderatoreffekte konnten dem Test nicht standhalten. Zudem liegen einige Unterschiede in der Stärke des Einflusses zwischen den Konstrukten des Bezugsrahmens vor.

Zunächst fällt auf, dass die Gesamteinstellung der IV-Verantwortlichen gegenüber dem Outsourcing am stärksten durch wahrgenommene Unterschiede in den Produktionskosten zwischen der Eigenerstellung und dem Fremdbezug beeinflusst wird. Dies belegt, dass Einsparungen in den Produktionskosten eines der wichtigsten Kriterien der Sourcingentscheidung darstellen (vgl. [Dibb ${ }^{+}$4]). Eine weitaus geringere Rolle spielen die Transaktionskosten (vgl. [AnSt98; Dibb ${ }^{+}$03]. Dies 
könnte darin begründet sein, dass Transaktionskosten im Gegensatz zu den Produktionskosten schwerer zu quantifizieren sind [Bart01]. Bemerkenswert erscheint, dass strategische Überlegungen eine durchaus wichtige Rolle bei der Sourcingentscheidung spielen. Dies bestätigt sowohl die Ergebnisse von Fallstudienuntersuchungen, die belegen, dass Unternehmen mit dem Outsourcing auch strategische Ziele verfolgen [DiRu98], als auch Arbeiten, die vor den strategischen Risiken des Outsourcings warnen [Ear196]. Interessante Ergebnisse liefert der Vergleich zwischen dem Einfluss dieser drei Faktoren auf die momentane Einstellung zum Outsourcing und den tatsächlichen Grad des Outsourcings. Wahrgenommene Unterschiede in den Produktionskosten und dem strategischen Beitrag zwischen der Eigenerstellung und dem Fremdbezug wirken sehr viel stärker auf die Gesamteinstellung als auf den momentan Grad des Outsourcings. Es besteht also eine Diskrepanz zwischen dem realen Sourcingverhalten und der momentanen Gesamtbeurteilung durch das IV-Management. Dies lässt darauf schließen, dass die mit der Sourcingentscheidung verbundenen ökonomischen und strategischen Zielvorstellungen nicht immer erreicht werden. Ins Bild passt auch, dass zwar ein positiver und signifikanter Zusammenhang zwischen den Einstellungen der IV-Leitung und dem Grad des Outsourcings vorliegt, dieser aber aufgrund der inhaltlichen Nähe der beiden Konstrukte hätte stärker ausfällen können. Offensichtlich ist die Einflussnahme des IV-Verantwortlichen auf das tatsächliche Sourcingverhalten begrenzt.

Besonders aufschlussreich sind allerdings die Umstände, unter denen die Eigenerstellung der Gestaltung und Wartung von Anwendungssoftware gegenüber dem Fremdbezug niedrigere Produktionskosten, Transaktionskosten sowie einen höheren strategischen Beitrag verspricht. Die Ergebnisse zeigen auf, dass die Eigenerstellung bei der Erreichung aller drei Zielgrößen Vorteile aufweist, wenn Anwendungssoftware-Dienstleistungen ein hohes Maß an spezifischen Investitionen in das Humankapital erfordern. Die Anwendungsgestaltung und -wartung ist dann besonders spezifisch, wenn ihre Verrichtung ein hohes Maß an sozialen Austauschbeziehungen zwischen den am Arbeitsprozess beteiligten Personen erfordert und ein hohes Maß an Wissen über unternehmensindividuelle Geschäftsprozesse notwendig ist. Etwas weniger wichtig ist das Wissen über unternehmensindividuelle Softwareanwendungen. Die Bedeutung der „user participation“ und der sozialen Interaktion wurde bereits in vielen Arbeiten als wichtiger Faktor bei der Entwicklung von Anwendungssystemen hervorgehoben (z. B. [Hirr $\left.\left.{ }^{+} 96\right]\right)$. Der tatsächliche ökonomische Einfluss dieser „,weichen Faktoren“ wurde bisher allerdings nur ungenügend untersucht. Insofern dürfen die zu Tage geförderten Ergebnisse auch als Beitrag zum besseren Verständnis der Produktions- und Kostenfunktion der Gestaltung und Wartung von Anwendungssoftware verstanden werden. Verstärkt wird dieser Eindruck noch durch den nachgewiesenen Einfluss zweier weiterer „weicher Faktoren“ auf Produktionskostenunterschiede. Es zeigt sich, dass auch interne Vorteile in der intrinsischen Motivation und der Vertrauenswürdigkeit der Mitarbeiter zu Kostenvorteilen der Eigenfertigung beitragen. Zudem zeigt sich, dass die Auslagerung spezifischer Anwendungssoftware-Dienstleistungen 
nicht unweigerlich zu erhöhten Transaktionskosten und dem Verlust strategischer Potenziale führt. Das Risiko erhöhter Transaktionskosten fällt dann besonders stark aus, wenn interne Mitarbeiter eine höhere intrinsische Motivation aufweisen. Das wahrgenommene strategische Risiko steigt, wenn interne Mitarbeiter als vertrauenswürdiger eingeschätzt werden. Werden hingegen Vorteile in der intrinsischen Motivation und der Vertrauenswürdigkeit beim Personal eines externen Dienstleisters gesehen, so verringern sich die Risken der Auslagerung spezifischer Anwendungssoftware. Als weiterer risikomindernder Faktor wirkt sich die Höhe der Kapitalbeteiligung am externen Dienstleister aus. Dies bestätigt die These, dass Eigentumsrechte die Gefahr opportunistischen Verhaltens mindern können. Folglich muss die Auslagerung von Anwendungssoftware-Dienstleistungen nicht zwingend mit einer erhöhten Standardisierung verbunden sein. Es kommt auch darauf an, wie die Outsourcingbeziehung konkret gestaltet wird und wie stark die Sozialkompetenz des Personals des externen Dienstleisters ausgeprägt ist.

Zusammenfassend lässt sich festhalten, dass es sich bei der Frage des Sourcings von Anwendungssoftware-Dienstleistungen um ein multidimensionales Entscheidungsproblem handelt. Unternehmen, die vor dieser Entscheidung stehen, sollten neben einer Kostenkalkulation, die sowohl die Produktions- als auch die Transaktionskosten einschließt, auch die strategischen Implikationen der Sourcingentscheidung berücksichtigen. Dabei empfiehlt es sich, die Anforderungen an die Gestaltung und Wartung von Anwendungssoftware vor dem Hintergrund der mit ihr verbundenen Zielsetzungen sorgfältig zu analysieren. Besonders wichtig erscheint es, zu ergründen, welche Arten von Wissen notwendig sind, wie dieses Wissen erzeugt wird, und welche Eigenschaften die internen oder externen Aufgabenträger aufweisen müssen, um die Zielvorgaben umzusetzen.

Obwohl die Ergebnisse durch eine fundierte statistische Analyse zu Tage gefördert wurden, gilt es zu berücksichtigen, dass es sich um keine tatsächlichen kausalen Zusammenhänge handelt. Es handelt sich vielmehr um eine Momentaufnahme des Sourcingverhaltens deutscher Unternehmen, aus der sich bestimmte Muster erkennen lassen. Auch die Verallgemeinerbarkeit der Ergebnisse ist eingeschränkt, da z. B. beim Modelltest nicht auf Branchen- und Funktionsunterschiede eingegangen wurde. Die Behebung dieser Limitationen bietet vielfältige Anknüpfungspunkte für zukünftige Studien. Des weiteren erscheint es zweckmäßig, den multitheoretischen Erklärungsansatz des IV-Outsourcings weiterzuverfolgen.

\section{Literatur}

[AjFi80] Ajzen, I.; Fishbein, M.: Understanding Attitudes and Predicting Social Behavior. Prentice Hall: Englewood Cliffs, NY, 1980.

[AlDe72] Alchian, A. A.; Demsetz, H.: Production, Information Costs, and Economic Organization. American Economic Review 62 (5), 1972, S. 777-795. 
[AnCu97] Ang, S.; Cummings, L. L.: Strategic Response to Institutional Influences on Information Systems Outsourcing. Organization Science 8 (3), 1997, S. 235-256.

[AnSt98] Ang, S.; Straub, D. W.: Production and Transaction Economies and IS Outsourcing: A Study of the U.S. Banking Industry. MIS Quarterly 22 (4), 1998, S. 535-552.

[Barn91] Barney, J. B.: Firm Resources and Sustained Competitive Advantage. Journal of Management 17 (1), 1991, S. 99-120.

[Bart01] Barthélemy, J.: The Hidden Costs of IT Outsourcing. Sloan Management Review Spring, 2001, S. 60-69.

[BeWa98] Beath, C. M.; Walker, G.: Outsourcing of Application Software: A Knowledge Management Perspective. Proceedings of the 31st Annual International Conference on System Sciences, Hawaii 1998, S. 666-674.

[Burr03] Burr, W.: Fundierung von Leistungstiefenentscheidungen auf der Basis modifizierter Transaktionskostenansätze. Zeitschrift für betriebswirtschaftliche Forschung 55 (März), 2003, S. 112-135.

[CaSt75] Calder, B. J.; Staw, B. M.: The Self-perception of Intrinsic and Extrinsic Motivation. Journal of Personality and Social Psychology 31, 1975, S. 599-605.

[Chin98] Chin, W. W.: The Partial Least Squares Approach for Structural Equation Modeling. In: Marcoulides, G. A. (Hrsg.): Modern Methods for Business Research. Lawrence Erlbaum Associates, Hillsdale 1998, S. 295-336.

[ChGo95] Chin, W. W.; Gopal, A.: Adoption Intention in GSS: Relative Importance of Beliefs. The DATA BASE for Advances in Information Systems 26 (2), 1995, S. 42-63.

[Chin ${ }^{+}$03] Chin, W. W.; Marcolin, B. L.; Newsted, P. R.: A Partial Least Squares Latent Variable Modeling Approach For Measuring Interaction Effects: Results From A Monte Carlo Simulation Study And Electronic Mail Emotion/Adoption Study. Information Systems Research 14 (2), 2003, S. 189-217.

[ClRo91] Clemons, E. K.; Row, M. C.: Sustaining IT Advantage: The Role of Structural Differences. MIS Quarterly 15 (3), 1991, S. 275-292.

[CoCo85] Couger, J. D.; Colter, M. A.: Maintenance Programming: Improved Productivity Through Motivation. Prentice Hall: Englewood Cliffs, NJ, 1985.

[Dibb ${ }^{+}$04] Dibbern, J.; Goles, T.; Hirschheim, R. A.; Jayatilaka, B.: Information Systems Outsourcing: A Survey and Analysis of the Literature. The DATA BASE for Advances in Information Systems forthcoming, 2004.

[Dibb $\left.{ }^{+} 01\right]$ Dibbern, J.; Güttler, W.; Heinzl, A.: Die Theorie der Unternehmung als Erklärungsansatz für das selektive Outsourcing der Informationsverarbeitung. Zeitschrift für Betriebswirtschaft 71 (6), 2001, S. 675-699.

[DiHe01] Dibbern, J.; Heinzl, A.: Outsourcing der Informationsverarbeitung im Mittelstand: Test eines multitheoretischen Kausalmodells. WIRTSCHAFTSINFORMATIK 43 (4), 2001, S. 339-350. 
[Dibb $\left.{ }^{+} 03\right]$ Dibbern, J.; Heinzl, A.; Leibbrandt, S.: Interpretation des Sourcing der Informationsverarbeitung: Hintergründe und Grenzen ökonomischer Einflussgrößen. WIRTSCHAFTSINFORMATIK 45 (5), 2003, S. 533-540.

[DiRu98] DiRomualdo, A.; Gurbaxani, V.: Strategic Intent for IT Outsourcing. Sloan Management Review Summer, 1998, S. 67-80.

[Ear196] Earl, M. J.: The Risks of Outsourcing IT. Sloan Management Review 37 (3), 1996, S. 26-32.

[FrOs97] Frey, B. S.; Osterloh, M.: Sanktionen oder Seelenmassage? Motivationale Grundlagen der Unternehmensführung. DBW 57 (3), 1997, S. 307-321.

[GoMo96] Ghoshal, S.; Moran, P.: Bad for Practice: A Critique of the Transaction Cost Theory. Academy of Management Review 21 (1), 1996, S. 13-47.

[GrHa86] Grossmann, S.; Hart, O.: The Costs and Benefits of Ownership: A Theory of Vertical and Lateral Integration. Journal of Political Economy 94, 1986, S. 671-719.

[Hein93] Heinzl, A.: Die Ausgliederung der betrieblichen Datenverarbeitung : eine empirische Analyse der Motive, Formen und Wirkungen. 2. Aufl., Poeschel: Stuttgart, 1993.

[Hirr ${ }^{+}$96] Hirschheim, R. A.; Klein, H. K.; Lyytinen, K.: Exploring the Intellectual Structures of Information Systems Development: A Social Action Theoretic Analysis. Accounting, Management and Information Technologies 6 (1/2), 1996, S. 1-64.

[HiLa00] Hirschheim, R. A.; Lacity, M. C.: The Myths and Realities of Information Technology Insourcing. Communications of the ACM 43 (2), 2000, S. 99-107.

[Ivia ${ }^{+}$02] Iivari, J.; Hirschheim, R.; Klein, H. K.: Towards More Professional Information Systems Development: ISD AS Knowledge Work. Proceedings of the 9th European Conference on Information Systems, Bled, Slovenia 2001.

[Laci ${ }^{+}$96] Lacity, M. C.; Willcocks, L. P.; Feeny, D. F.: The Value of Selective IT Sourcing. Sloan Management Review 37 (3), 1996, S. 13-25.

[PiMa92] Picot, A.; Maier, M.: Analyse- und Gestaltungskonzepte für das Outsourcing. Information Management 4, 1992, S. 14-27.

[Pint $\left.{ }^{+} 93\right]$ Pinto, M. B.; Pinto, J. K.; Prescott, J. E.: Antecedents and Consequences of Project Team Cross-functional Cooperation. Management Science 39 (10), 1993, S. 12811297.

[Ragh99] Raghunathan, B.; Raghunathan, T. S.; Tu, Q.: Dimensionality of the Strategic Grid Framework: The Construct and its Measurement. Information Systems Research 10 (4), 1999, S. 343-355.

[Will81] Williamson, O. E.: The Economics of Organization: The Transaction Cost Approach. American Journal of Sociology 87 (3), 1981, S. 548-577.

[ZaVe94] Zaheer, A.; Venkatraman, N.: Determinants of Electronic Integration in the Insurance Industry: An Empirical Test. Management Science 40 (5), 1994, S. 549-566. 